\title{
THE OCTOBER MEETING IN NEW HAVEN
}

The four hundred eighty-fourth meeting of the American Mathematical Society was held at Yale University on Saturday, October 25, 1952, in the Osborn Zoological Laboratory. There were about 200 persons in attendance including the following 169 members of the Society:

C. R. Adams, R. B. Adams, D. B. Ames, Joseph Andrushkiw, W. G. Bade, R. B. Barrar, J. Y. Barry, R. G. Bartle, G. E. Bates, J. D. Baum, Anatole Beck, E. G. Begle, P. H. Belisle, R. R. Bernard, Benjamin Bernholtz, D. L. Bernstein, L. F. Boron, C. J. Bouwkamp, J. W. Bower, Richard Brauer, F. E. Browder, A. B. Brown, A. R. Brown, L. W. Cohen, H. S. Collins, R. M. Conkling, T. F. Cope, V. F. Cowling, J. B. Crabtree, Frederic Cunningham, M. D. Darkow, M. D. Davis, R. B. Davis, Allen Devinatz, D. J. Dickinson, H. L. Dorwart, T. C. Doyle, G. R. Duke, Nelson Dunford, W. L. Duren, William H. Durfee, Aryeh Dvoretsky, J. E. Eaton, Samuel Eilenberg, Joanne Elliott, Trevor Evans, J. M. Feld, Chester Feldman, William Feller, W. E. Ferguson, Alvaro Ferlini, R. M. Foster, W. C. G. Fraser, J. B. Freier, Bernard Friedman, M. P. Gaffney, Murray Gerstenhaber, H. A. Giddings, Oscar Goldman, L. W. Green, Laura Guggenbuhl, F. C. Hall, Duncan Harkin, Melvin Hausner, G. A. Hedlund, Henry Helson, S. B. Hobbs, S. P. Hoffman, Alfred Horn, W. A. Howard, J. L. Howell, D. H. Hyers, H. G. Jacob, Nathan Jacobson, R. E. Johnson, R. V. Kadison, Shizuo Kakutani, Aida Kalish, M. E. Kellar, Fred Kiokemeister, George Klein, W. J. Klimczak, A. T. Kovitz, P. H. Kratz, Serge Lang, Solomon Leader, Jean Leray, Marie Lesnick, D. J. Lewis, W. G. Lister, A. E. Livingston, Eugene Lukacs, B. H. McCandless, G. W. Mackey, H. M. MacNeille, L. F. Markus, H. G. Mazurkiewicz, L. F. Meyers, E. J. Miles, W. H. Mills, Don Mittleman, R. E. Montgomery, J. C. Moore, Morris Morduchow, G. D. Mostow, Simon Mowshowitz, D. J. Newman, E. N. Nilson, Katsumi Nomizu, E. T. Onat, R. H. Owens, F. P. Pedersen, F. W. Perkins, R. S. Pierce, H. O. Pollak, Harry Pollard, M. H. Protter, F. A. Quigley, R. A. Raimi, Helene Reschovsky, H. G. Rice, C. E. Rickart, R. A. Roberts, G. B. Robison, Hartley Rogers, I. H. Rose, J. P. Russell, Charles Salkind, J. E. Sammet, Arthur Sard, W. K. Saunders, R. D. Schafer, Robert Schatten, Abraham Schwartz, J. T. Schwartz, C. H. W. Sedgewick, C. B. Seligman, V. L. Shapiro, O. K. Smith, N. E. Steenrod, Marvin Stern, R. L. Sternberg, F. M. Stewart, M. H. Stone, Walter Strodt, M. M. Sullivan, R. L. Taylor, D. L. Thomsen, J. I. Tracey, H. G. Tucker, Bryant Tuckerman, D. F. Votaw, J. V. Wehausen, H. F. Weinberger, Morris Weisfeld, Paul Weiss, David Wellinger, John Wermer, A. L. Whiteman, Hassler Whitney, Albert Wilansky, C. H. Wilcox, A. B. Willcox, N. Z. Wolfsohn, K. G. Wolfson, Arthur Wouk, Fumio Yagi, D. M. Young, Oscar Zariski.

An address The linear hyperbolic differential equation, was presented at the General Session by Professor Jean Leray of the Collège de France and The Institute for Advanced Study by invitation of the Committee to Select Hour Speakers for Eastern Sectional Meetings. Professor William Feller presided.

Sessions for contributed papers were held in the morning, Professor W. L. Duren presiding, and in the afternoon, Professor T. F. Cope presiding. 
Abstracts of the papers presented at the meeting follow, those with " $t$ " after the abstract number having been read by title. Of the papers having joint authorship paper 4 was presented by Dr. Taylor, paper 11 by Professor Cowling, and paper 12 by Dr. Weinberger. Mr. Chehata was introduced by Dr. B. H. Neumann, Professor Zassenhaus by Professor S. A. Jennings, and Mrs. Dickinson by Professor R. L. Wilder.

\section{Algebra AND Theory of Numbers}

\section{1t. Leonard Carlitz: Some sums analogous to Dedekind sums.}

Analogues of the Dedekind sums are constructed in the field $G F(q, x)$. The most interesting property of the analogues is a reciprocity theorem analogous to the well known Dedekind reciprocity theorem. In addition analogues are obtained for Theorems 1 and 2 of Rademacher and Whiteman's paper (Amer. J. Math. vol. 63 (1941) pp. 377-407). (Received August 11, 1952.)

\section{2t. Leonard Carlitz: The reciprocity theory for Dedekind sums.}

By means of the representation $s(h, k)=1 / 4 k+(1 / k) \sum_{\zeta \neq 1}\left(\zeta^{-1}-1\right)^{-1}\left(\zeta^{h}-1\right)^{-1}$, where $\zeta$ runs through the $k$ th roots of unity and $s(h, k)$ denotes the Dedekind sum, a proof of the reciprocity theorem is given. The proof was suggested by Redei's proof (Acta Scientarium Mathematicarum vol. 12, part B (1950) pp. 236-239). The method also applies to Apostol's generalization (Duke Math. J. vol. 17 (1950) pp. 147157). (Received August 11, 1952.)

\section{3t. C. G. Chehata: On an ordered semigroup.}

The semigroup of Malcev (Math. Ann. vol. 113 (1937) pp. 686-691), which is known not to be embeddable in a group, can be turned into a (fully) ordered semigroup. This answers negatively the question whether an ordered semigroup can always be embedded in a group, ordered or not. An incidental consequence is that the ring considered by Malcev (loc. cit.), which can not be embedded in a division ring, can nevertheless be embedded in a formal power series ring. (Received September 8, 1952.)

\section{Anne P. Cobbe and R. L. Taylor: On Q-kernels with operators.}

Let $H$ be an aditive abelian group with a multiplicative group of operators $B$. A domain for $(B, H)$ is a set $D$ with an additive, simply transitive operation of $H$ on $D$ and a multiplicative operation of $B$ on $D$, such that $b(h+d)=b h+b d$. The isomorphism-classes of domains form a group, isomorphic in a natural way to $H^{1}(B, H)$. The deviation of a domain $D$ is the corresponding element of $H^{1}(B, H)$; it is zero if and only if $D$ has a fixed point under $B$. Let $\theta: Q \rightarrow A(K) / I(K)$ be an extendible $Q$-kernel; let $B$ denote the group of all automorphisms $b$ of $Q$ such that $\theta b=\theta$. If $(E, \rho)$ is an extension of $\theta: Q \rightarrow A(K) / I(K)$, then so is $(E, b \rho)$, for every $b \in B$. Thus $B$ operates on the set $\mathcal{E}$ of all isomorphism-classes of extensions of $\theta: Q \rightarrow A(K) / I(K) . B$ also operates on $H^{2}\left(Q, Z_{K}\right) ; \mathcal{E}$ forms a domain for $\left[B, H^{2}\left(Q, Z_{K}\right)\right]$, whose deviation $\in H^{1}[B$, $\left.H^{2}\left(Q, Z_{K}\right)\right]$ is called the domain-deviation of $\theta: Q \rightarrow A(K) / I(K)$. An example shows

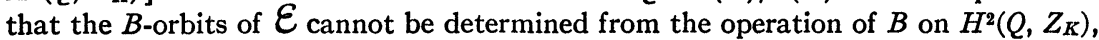
without knowledge of the domain-deviation; the latter is computed explicitly for cyclic $Q$. The equivariant obstruction, an element of $H_{e}^{3}\left(Q, Z_{K}\right)$, is defined whether 
$\theta: Q \rightarrow A(K) / I(K)$ is extendible or not; it determines, but is not determined by, the Eilenberg-MacLane obstruction; in the extendible case, it determines, but is not determined by, the domain-deviation. The ideas are generalized to the case of a $Q$-kernel with a group $B$ operating on both $Q$ and $K$. (Received August 5, 1952.)

\section{Serge Lang: The theory of real places.}

Let $K$ and $L$ be two fields. An $L$-valued place $\phi$ of $K$ is a homomorphism of $K$ into $\{L, \infty\}$. We say that $\phi$ is a real place if $L$ is a real field. If $\phi$ is a real place of $K$ taking its values in a real closed field $R$, then $K$ is real and $\phi$ can be extended to an $R$-valued place of a real closure of $K$. The real places of $K$ can be completely classified in terms of the possible orderings of $K$. Let $K$ be a function field (in several variables) over a real constant field $F$ and suppose $K$ is ordered. Let $\xi_{1}<\xi_{2}<\cdots<\xi_{n}$ be a finite set of elements of $K$. Then there exists a zero-dimensional real place $\phi$ of $K$ (i.e. $\phi$ takes its values in the real closure $R$ of $F$ and is identity on $F$ ) such that all $\phi\left(\xi_{i}\right)$ are finite and $\phi\left(\xi_{1}\right)<\phi\left(\xi_{2}\right)<\cdots<\phi\left(\xi_{n}\right)$. This generalizes the Hilbert Problem solved by Artin. Sturm's Theorem remains an essential step in the argument. (Received July 30,1952 .)

\section{6t. G. N. Raney: A subdirect-union representation for completely dis-} tributive complete lattices. Preliminary report.

Let $L$ be a complete lattice. Define a semi-ideal of $L$ to be a subset $M$ of $L$ such that if $y \in M$ and $x \leqq y$, then $x \in M$. For every $y \in L$, let $K(y)$ be the intersection of the family of semi-ideals $M$ such that $\bigcup M \geqq y$. It is shown that $L$ is completely distributive if and only if for every $y \in L, \bigcup K(y)=y$. Let $x \rho y$ mean $x \in K(y)$; then the relation $\rho$ has the property that $\rho \circ \rho=\rho$. Moreover, it is shown that with every relation $\rho$ such that $\rho \circ \rho=\rho$ there is associated a competely distributive complete lattice $L$, in such a way that if $\rho$ is reflexive (and hence a quasi-ordering) then $L$ is isomorphic with a complete ring of sets. This generalizes a result of $\mathrm{G}$. Birkhoff. In terms of these notions it is proved that every completely distributive complete lattice is isomorphic with a closed sublattice of the direct union of a family of complete chains. (Received September 5, 1952.)

\section{7t. H. J. Zassenhaus: On the representations of Lie algebras of prime characteristic.}

It is shown that the enveloping algebra $A(L)$ of a Lie algebra $L$ of finite dimension $n$ over a field $F$ of prime characteristic $p$ is a maximal order of a division algebra $K$ of dimension $p^{2 m}$ over its center $Q(Z)$, the quotientfield of the center $Z$ of $A(L) . Z$ is an algebraic variety of dimension $n$. Each specialization $\Theta$ of $Z$ onto $F$ over $F$ is extendible to a specialization $\Theta^{*}$ of $A(L)$ onto a hypercomplex system $\Theta^{*} A(L)$ over $F$. Each absolutely irreducible representation $\Delta^{*}$ of $\Theta^{*} A(L)$ over $F$ induces the absolutely irreducible representation $\Delta$ of $L$ given by $\Delta(a)=\Delta^{*} \Theta^{*}(a)$ and each absolutely irreducible representation of $L$ is obtained this way. The degree of $\Delta$ is not greater than $p^{m}$ and smaller than $p^{m}$ only for the genuine subvariety of specializations of $Z$ mapping the discriminant ideal of $A(L)$ over $Z$ onto zero. The absolutely irreducible constituents of an absolutely indecomposable representation of $L$ over $F$ all belong to the same specialization $\Theta$ of $Z$ onto $F$. For each $\Theta$ there are absolutely indecomposable representations of arbitrary high degree. Application is made to nilpotent Lie algebras and the Lie algebras of Witt where some of the results had been known before. (Received September 8, 1952.) 


\section{ANALYsis}

\section{Joseph Andrushkiw: On the positivity of a polynomial for posi- tive values of the variable.}

Let $z_{1}, z_{2}, \cdots, z_{n}$ denote the zeros of the real polynomial $f(z), s_{k}=\sum_{j=1}^{k} z_{j}^{k}$, the $D_{k}$ and $\bar{D}_{k}$ the determinants $\left|s_{i+j}\right|, i, j=0,1, \cdots, k-1$, and $\left|s_{i+j-1}\right|, i, j=1,2$, $\cdots, k$, respectively. Denoting by $d$ and $\bar{d}$ the differences $p-v$ and $\bar{p}-\bar{v}$ of the sign permanences and sign variations in the sequences $1, D_{1}, \cdots, D_{n}$ and $1, \bar{D}_{1}, \cdots, \bar{D}_{n}$ respectively, one obtains, following the method of Hermite (E. Netto, Vorlesungen ueber Algebra, vol. 1, p. 263-270), that $d+\bar{d}=0$ is necessary and sufficient condition that $f(z)>0, z \geqq 0$, in case, if $D_{k} \neq 0$ and $\bar{D}_{k} \neq 0, k=1,2, \cdots, n$ (regular case). Based on the results obtained in a former paper (Bull. Amer. Math. Soc. Abstract 58-3-289) another criterion, without any restriction, can be obtained. Putting $f\left(-z^{2}\right)=g(z)$, $F(z)=g(z+x)$, the Hurwitzian determinant (Hurwitz, Werke, vol. 2, p. 534) $H_{2 n}(x)$ of $F(z)$ is constructed. Let $H_{j}^{(b)}(x)$ denote the th derivative of its principal minor of order $j$. If $H_{j}(0)=H_{j}^{\prime}(0)=\cdots=H_{j}^{(L-1)}(0)=0, H_{j}^{(\iota)}(0)=h_{j} \neq 0$, let $v$ denote the number of sign variations in the sequence $1, h_{1}, h_{2} / h_{1}, \cdots, h_{2 n} / h_{2 n-1}$. Then $v=n$ is necessary and sufficient condition that $f(z)>0, z \geqq 0$. In the regular case it is shown that $h_{2 k-1}=D_{k}, h_{2 k}=\bar{D}_{k}$. (Received September 10, 1952.)

9. F. E. Browder: Completeness of eigenfunctions and eigenfunction expansions for non self-adjoint linear elliptic differential equations of arbitrary order.

Let $K$ be a suitably differentiable linear elliptic differential operator of order $2 m$ on the bounded domain $D$ of $E^{n}, B$ a suitably differentiable positive self-adjoint differential operator on $D$ of order less than $2 m$. If $\phi \in C^{2 m}(D)$ and vanishes with all its derivatives of order less than $m$ on the boundary of $D, \phi$ is said to be an $n$th order eigenfunction of $K$ with respect to $B$ and with $B$-eigenvalue $\lambda$ provided that $K \phi-\lambda B \phi$ $=B \psi$ where $\psi$ is an eigenfunction of order less than $n$ of $K$ with respect to $B$ and with $B$-eigenvalue $\lambda$. For $n=1$ these are the ordinary eigenfunctions of $K$ with respect to $B$. It is shown that there exists a set of eigenfunctions of $K$ with respect to $B$ which is complete and forms a basis in $L^{2}(D)$ and $H_{m}(D)$. If $K$ commutes with $B$, the eigenfunctions of order $n$ satisfy the equation $(K-\lambda B)^{n} \phi=0$. Use is made in the proofs of the operational calculus for analytic functions of linear operators on a Banach space developed by Gelfand, Lorch, and Dunford. (Received September 11, 1952.)

10. H. S. Collins: Completeness and weak compactness in linear topological spaces.

Let $X$ be a locally convex Hausdorff linear topological space over the reals $R$. $X$ has property A [property B] if the bounded weak-* and weak-* closures for linear [maximal linear] subspaces of $X^{*}$ coincide. A set $M$ in $X$ is mean compact if given any sequence of points $\left\{x_{n}\right\}$ in $X$ there is a point $x$ in $X$ such that $\lim \inf _{n} f\left(x_{n}\right)$ $\leqq f(x) \leqq \lim \sup _{n} f\left(x_{n}\right)$ for all $f$ in $X^{*}$. The results are: 1 . If $M$ is mean compact, then $\bar{M}$ is contained in the adjoint of $\left(X^{*}\right.$, bounded weak-*), where $\bar{M}$ denotes the closure of $M$ in the product topology of $R^{X^{*}}$. If $X$ has property $\mathrm{B}$, it follows that mean compactness and the various well known weak compactness notions are all equivalent, a result which generalizes earlier work of Smulian, Phillips, and Eberlein, and later work of Grothendieck. 2. Property B is equivalent to completeness. 3. Property A is, in general, stronger than property $\mathrm{B}$, and if $X$ has property $\mathrm{A}$ and $L$ is any closed 
linear subspace of $X$, then $L, X / L$, any linear-open image of $X$, and any finer topology for $X$ giving the same adjoint all have property A. In virtue of these results, property A is suggested as a more desirable definition of completeness in locally convex spaces. (Received May 26, 1952.)

\section{V. F. Cowling and W. J. Thron: Zero-free regions of polyno-} mials.

In this paper we obtain certain results about the location of the zeros of a polynomial. We use a method which was developed by Leighton and Thron (Bull. Amer. Math. Soc. vol. 50) to study the convergence of continued fractions and was then adapted by Cowling (Proceedings of the American Mathematical Society vol. 2) to obtain results on the distribution of values of the partial sums of a Taylor series. This latter method has now been further modified to make it as suitable as possible for our present purpose. A typical theorem which is proved is the following: Let $P(z)=a_{0}+a_{1} z^{r_{1}}+\cdots+a_{n} z^{r_{n}}$ where all $a_{k}, k=0, \cdots, n$, are different from zero and where $r_{1}<r_{2}<\cdots<r_{n}$. Then $P(z)$ assumes all its zeros for $|z| \leqq \max _{1 \leqq k \leqq n}\left(\left(\left(1+r_{k-1}\right) / r_{k}\right)\right.$ $\left.\left|a_{k-1} / a_{k}\right|\right)^{1 /\left(r_{k}-r_{k-1}\right)}$ where $r_{0}=0, r_{n}=1$, and the remaining $r_{k}$ are arbitrary positive numbers. A well known result (see Dieudonné, La therorie analytique des polynomes d'une variable, p. 18) is obtained as a corollary of this theorem if we set $\gamma_{k}=1, k=1, \cdots, n$. (Received August 15, 1952.)

12. J. B. Diaz and H. F. Weinberger: $A$ solution of the singular initial value problem for the Euler-Poisson-Darboux equation.

A solution is found for the initial value problem $\left(^{*}\right) \Delta u=u_{t t}+(K / t) u_{t}$ with $u\left(x_{1} \cdots x_{m}, 0\right)=f\left(x_{1}, \cdots, x_{m}\right), u_{t}\left(x_{i}, 0\right)=0$ where $K$ is a real constant and $f$ is given. (Another solution for this initial value problem was recently given by A. Weinstein, C. R. Acad. Sci. Paris vol. 234 (1952) pp. 2584-2585.) It is based on the known fact that for $K=m-1$, the solution of $\left(^{*}\right)$ is $M\left(x_{i}, t ; f\right)$, the mean value of $f$ over the surface of the sphere of radius $t$ about the point $\left(x_{1}, \cdots, x_{m}\right)$. For $K>m-1$, the solution of $\left(^{*}\right)$ is given by an integral formula which coincides with the solution obtained by Hadamard's method of descent for $K=m, m+1, m+2, \cdots$. For $K \leqq m-1$, this integral does not converge, but the function of $K$ represented by it is continued analytically. In this manner, the following solution of $\left({ }^{*}\right)$ is obtained for $K>m-2 p-3(p$ a non-negative integer, $K \neq-1,-3, \cdots): A \int_{0}^{1} N_{p}\left(x_{i}, \alpha, t ; f\right)$ $\left(1-\alpha^{2}\right)^{(K-m-1) / 2} \alpha^{m-1} d \alpha+B \sum_{n=0}^{p}\left(t^{n} / n !\right)\left(\partial^{n} M / \partial t^{n}\right)\left(x_{i}, t ; f\right) \cdot S_{n}$ where $A, B$, and $S_{n}$ are known constants, and $N_{p}$ is $M\left(x_{i}, \alpha t ; f\right)$ minus the first $p+1$ terms of its Taylor series in $\alpha$, about $\alpha=1$. For $m-k$ a positive odd integer, the first term disappears and one obtains a Huygens' principle. (Received September 8, 1952.)

\section{Joanne Elliott: The boundary value problems and semi-groups} associated with certain integro-differential operators.

We are concerned with initial value problems for the pair of equations (1) $u_{t}(x, t)$ $=\pi^{-1} P \cdot \int_{-1}^{+1}\left(u_{\xi}(\xi, t) /(\xi-x)\right) d \xi=\Omega u$ and $(2) u_{t}(x, t)=\pi^{-1}(d / d x) P \cdot \int_{-1}^{+1}((u(\xi, t) /(\xi-x)) d \xi$ $=\Omega^{*} u$ in $C[-1,+1]$ and $L[-1,+1]$ respectively. Probabilistically, these equations bear the same relation to the Cauchy process as the backward and forward heat equations on a finite interval bear to homogeneous diffusion on the infinite interval. Hence, it is surprising that each equation behaves analytically like a certain parabolic differential equation with non-constant coefficients. The solutions are treated as semi-groups of transformations on the initial values. Following the method of Feller 
(Ann. of Math. vol. 55), we start with (1) and study the resolvent equation $\lambda f-\Omega f=h$ in $C[-1,+1]$. This is reduced to an integral equation, and the existence of positive, norm-not-increasing solutions is proved. It is shown, by use of the Hille-Yosida criterion for infinitesimal generators, that the most general boundary conditions for the parabolic equations have analogues in this case. The adjoint semi-groups are then studied, and it is shown that in certain important cases they are generated by $\Omega^{*}$, but that in general the generators will contain additional terms. (Received September 10, 1952.)

\section{4t. William Feller: On the generation of strongly continuous semi- groups.}

Let $\left\{T_{t}\right\}$ be a semi-group of bounded linear operators from a Banach space $X$ to itself which is (strongly measurable and therefore) strongly continuous for $t>0$. Under the restrictive conditions (1) $T_{t} x \rightarrow x$ as $t \rightarrow 0$ for each $x \in X$, (2) $\left\|T_{t}\right\|<1+c t$ for $t<1$, Hille and Yosida defined an infinitesimal generator $\Omega$ and succeeded in characterizing all infinitesimal generators in terms of the resolvent $(\lambda I-\Omega)^{-1}$. In this paper an analogous result is obtained for the general case where both (1) and (2) are dropped. Then $\left\|T_{t}\right\|$ may be unbounded near $t=0$. The theory of the "resolvent" goes through although $(\lambda I-\Omega)^{-1}$ is an unbounded operator and the entire right half of the $\lambda$-plane may belong to the continuous spectrum of $\Omega$. By specialization one obtains a characterization of $\Omega$ for the case of strong continuity at $t=0$ (condition 1). (Received September 10, 1952.)

\section{5t. William Feller: Semi-groups of transformations in general weak} topologies.

For semi-groups $\left\{T_{t}\right\}$ of bounded linear operators from a real Banach space $X$ to itself usually strong measurability is assumed. Translations in $B V(-\infty,-\infty)$ are not even weakly measurable, translations in $C(-\infty,-\infty)$ not strongly measurable. A more general approach avoids these difficulties. Let $X^{*}$ be the conjugate space of $X$ and $T_{t}^{*}$ the adjoint transformation to $T_{t}$. Let $Y \subset X^{*}$ be invariant under $\left\{T_{t}^{*}\right\}$ (e.g. $Y=$ subspace generated by $T_{t}^{*} x_{0}^{*}$ ). Call $\left\{T_{t}\right\}$ weakly $(Y)$ measurable if the real function $x^{*}\left(T_{t} x\right)$ is $L$-measurable whenever $x \in X, x^{*} \in Y$. Considering $y \in Y$ as operator from $X$ to the set of $L$-measurable functions leads to a definition of "essential norm" $\left\|T_{t}\right\|_{e}$. It is $L$-measurable and bounded in $\delta<t<\delta^{-1}$ (while, according to $\mathrm{R}$. S. Phillips, neither statement is true of $\left\|T_{t}\right\|$, even if $\left\{T_{t}\right\}$ is weakly measurable, i.e. $\left.Y=X^{*}\right)$. It is shown that $\left\{T_{t}\right\}$ can be extended to spaces $X_{e} \supset X, Y_{0} \supset Y$ in which $\left\{T_{t}\right\}$ is closed under Pettis integration, and has a weak continuity property expressible in terms of Banach's transfinite limits. Each of these spaces contains a subspace in which $\left\{T_{t}\right\}$ is strongly continuous with a norm $\leqq\left\|T_{t}\right\|_{e .}$. The notions of infinitesimal generator and resolvent are generalized. Let $X_{0}$ be the space of those $x \in X$ for which $y T_{t} x=0$ a.e. for each $y \in Y$. The quotient space $x / X_{0}$ can be remetrized and completed under a finer topology so as to make $\left\{T_{t}\right\}$ weakly continuous with bounded true norm. (Received September 10, 1952.)

16t. A. E. Livingston: The Lebesgue constants for the Euler methods of summability.

The Euler method $(E, t), 0<t \leqq 1$, is defined by the infinite matrix $\left(a_{n k}\right)$ where $a_{n k}={ }_{n} C_{k} t^{k}(1-t)^{n-k}$ for $k=0, \cdots, n$ and $a_{n k}=0$ for $k=n+1, n+2, \cdots$. The Lebesgue 
constants for the method $(E, t)$ are then given by $L_{n}(t)=(2 / \pi) \int_{0}^{\pi}\left(\mid \operatorname{Im}\left[e^{i x / 2}(1-t\right.\right.$ $\left.\left.\left.+t e^{i x}\right)^{n}\right] \mid / 2 \sin 2^{-1} x\right) d x$. The author shows that $L_{n}(t)=\left(2 / \pi^{2}\right) \log n+O(1)$ for $0<t<1$. (Received October 25, 1952.)

17t. G. W. Mackey: A Frobenius reciprocity theorem for noncompact subgroups. Preliminary report.

Let $G$ be a separable unimodular locally compact group and let $G$ be a unimodular closed subgroup of $G$. Let the regular representations of $G$ and $G$ be of type I and let their canonical decompositions into factor representations be $\int_{X}^{x} F d \zeta(x)$ and $\int_{Y}^{y} H d \eta(y)$ where ${ }^{x} F$ is a multiple of the irreducible representation ${ }^{x} L$ of $G$ and ${ }^{u} H$ is a multiple of the irreducible representation ${ }^{\nu} M$ of $G$. Then there exist functions $x \rightarrow \beta_{x}$ and $y \rightarrow \gamma_{y}$ where each $\beta_{x}$ is a measure on $Y$ and each $\gamma_{y}$ is a measure on $X$ such that the measures $\int \gamma_{\nu} d \eta(y)$ and $\int \beta_{x} d \zeta(x)$ on $X \otimes Y$ are identical. Moreover if $\alpha$ denotes this measure, then there exists an $\alpha$ measurable function $n$ from $X \otimes Y$ to the at most countable cardinals such that for almost all $x$ the representation of $G$ induced by ${ }^{x} L$ is equivalent to $\int_{Y} n(x, y)^{y} M d \beta_{x}(y)$ and for almost all $y$ the restriction to $G$ of ${ }^{y} M$ is equivalent to $\int_{X} n(x, y)^{x} L d \gamma_{y}(x)$. When $G$ is compact the result takes on a simpler form and the hypothesis on the regular representation of $G$ can be omitted. This case has been treated by Mautner by what seem to be rather different methods. The hypothesis of unimodularity can be weakened and probably eliminated altogether. (Received September 10, 1952.)

\section{8t. J. L. Massera: Total stability and approximately periodic vibra-} tions.

Let $\dot{x}=X(x, t), X(0, t)=0$, be a system of differential equations. The solution $x=0$ is said to be totally stable if given $\epsilon>0$ there is a $\delta>0$ such that the solutions $x(t)$ of a varied system $\dot{x}=X_{1}$ satisfy $\|x(t)\|<\epsilon$ for $t \geqq 0$, provided that $\|x(0)\|<\delta$ and $\left\|X-X_{1}\right\|<\delta$. A sufficient condition for the total stability is the existence of a Lyapunov function $V(x, t)$ with the properties: (a) $V$ is positive definite; (b) $d V / d t$ is negative definite; (c) the partials of $V$ are bounded. It is possible to prove similar sufficient conditions for the total stability of a solution in the "orbital" sense. A function $x(t)$ is said to be $\epsilon^{+}$-periodic of period $T$ if there is a sequence $t_{n}, t_{0}=0,0<T-\epsilon$ $<t_{n+1}-t_{n}<T+\epsilon$, such that $\left|x\left(t_{n}+\tau\right)-x(\tau)\right|<\epsilon$ for $0 \leqq \tau \leqq t_{n+1}-t_{n}$. It is then possible to show that systems of differential equations which are close to systems having periodic totally stable (in the strong or in the orbital sense) solutions, have $\epsilon^{+}$-periodic solutions. (Received September 15, 1952.)

\section{M. H. Protter: The Cauchy problem with data on the parabolic line.}

Consider the equation $K(y) P(x, y) u_{x x}-u_{y y}+a(x, y) u_{x}+b(x, y) u_{y}+c(x, y) u$ $=f(x, y)$ where $K(y)$ behaves like $y^{\alpha}, \alpha>0$, in a neighborhood of $y=0, P(x, y)>0$ for all $x, y$, and all coefficients are, say, twice differentiable. The Cauchy problem for the above equation is treated when the initial data are prescribed along a segment of the $x$-axis (which is a parabolic line). It is shown that the Cauchy problem is correctly set if $y a(x, y) /(K(y))^{1 / 2} \rightarrow 0$ as $y \rightarrow 0$. This generalizes previous results of Berezin and Bers for the Cauchy problem. The method consists of reducing the above equation to a system of the first order and then solving the corresponding system of integral equations obtained by integrating along characteristics. (Received September 10, 1952.) 


\section{V. L. Shapiro: An extension of results in the uniqueness of double trigonometric series.}

Let $T=\sum a_{m n} e^{i(m x+n y)}$ be a double trigonometric series whose coefficients $a_{m n}$ $=o(1)$. Call $T$ a series of type (U) if the circular partial sums of the series $\sum\left(a_{m n} /\left(m^{2}+n^{2}\right)\right) e^{i(m x+n y)}$ converge uniformly. Suppose the circular partial sums of $T$ are $(C, 1)$ summable to a function $f(x, y)$. It is known (M. Cheng, Ann. of Math. vol. 52 (1950) pp. 403-416) that if $f(x, y)$ is mean continuous everywhere and the Fourier series of $f(x, y)$ is of type (U), then $T$ is the Fourier series of $f(x, y)$. In this paper it is proved that $T$ is also the Fourier series of $f(x, y)$ if $f(x, y)$ is an essentially bounded function. In case $T$ is a double sine series of type (U), then it is shown in this paper that $f(x, y)$ need only be an integrable function for $T$ to be its Fourier series. (Received September 10, 1952.)

\section{1t. Bertram Yood: Complex Banach algebras with an involution.}

Let $B, B_{1}$ be two complex Banach algebras with an involution. Call $B$ a $\rho^{*}$-algebra if there exists an auxiliary norm $|x|$ (in which $B$ is a not necessarily complete normed algebra) and a number $k>0$ such that $\rho(x) \geqq k|x|$ for all self-adjoint (s.a.) $x$, where $\rho(x)$ is the spectral radius of $x$. Call $B$ a $\rho_{a}^{*}$-algebra if the auxiliary norm can be chosen with $B$ complete in it. An important distinction between these algebras and the algebras with involution usually treated is that $x^{*} x=0$ does not imply $x=0$. It is shown that if $B$ is a $\rho^{*}$-algebra in which every s.a. element has real spectrum, then $B$ has the uniqueness of norm property discussed by Rickart [Ann. of Math. vol. 51 (1950) pp. 615-628]. If $B$ and $B_{1}$ are two $\rho_{0}^{*}$-algebras where every s.a. element in $B$ has real spectrum and the involution in $B_{1}$ is continuous, then any algebraic *-isomorphism of $B$ into $B_{1}$ is bi-continuous. Conditions are given under which a $\rho_{c}^{*}$-algebra is ${ }^{*}$-isomorphic to a $C^{*}$-algebra where the *-isomorphism is bi-continuous. (Received September 10, 1952.)

\section{2t. Bertram Yood: Homomorphisms defined on regular Banach} algebras.

Let $T$ be an algebraic homomorphism of a complex commutative Banach algebra $B_{1}$ into another such algebra $B_{2}$. It is assumed that each $B_{i}$ has an identity element $e_{i}$, $T\left(e_{1}\right)=e_{2}$, and that $B_{1}$ is regular in the sense of Silov [Trav. Inst. Math. Stekloff vol. 21 (1947) ]. It is shown that $\overline{T\left(B_{1}\right)}$ is always regular, and that for each maximal ideal $M, T(M)$ is contained in a maximal ideal of $B_{2}$ if and only if $T^{-1} \overline{(T(R))}=R$ where $R$ is the radical of $B_{1}$. If $B_{1}$ is semi-simple, $T$ one-to-one, and the mapping $x \rightarrow x(M)$ of $B_{1}$ into the algebra of continuous functions on its space of maximal ideals has closed range, then $T$ is continuous and $T\left(B_{1}\right)$ is closed. Some results are also obtained for the situation where $T$ is not an isomorphism. (Received September 12, 1952.)

23t. H. J. Zimmerberg: On the Green's matrix of a differential system.

Let $G(x, t)$ be the Green's matrix for an incompatible homogeneous differential system $\mathcal{L}[y] \equiv y^{\prime}-A(x) y=0, M y(a)+N y(b)=0, M$ and $N$ complex constant matrices such that the boundary conditions are linearly independent. A theorem of Miller and Schiffer [Proceedings of the American Mathematical Society vol. 3 (1952) p. 436] is extended to show how $n$ of the columns of the $n \times 2 n$ matrix $\|G(x, a) G(x, b)\|$ 
may be selected as the columns of a fundamental matrix solution of $\mathcal{L}[y]=0$. (Received August 21, 1952.)

\section{Applied Mathematics}

24. T. C. Doyle: Topological and dynamical invariant theory of an electrical network.

If $s$ is a row matrix of the $n$ oriented segments of a network $G$, the expression $g_{i}=s \xi_{i}$, $\xi_{i}$ a column matrix of integers, is interpreted as an oriented subgraph $g_{i}$ whose segment $s_{k}$ has multiplicity $\xi^{k}$. Any transformation $g=s \xi$ of an infinite group $G_{\xi}$ is regarded as a change of basis in the vector space $V_{g}$ of subgraphs from the primitive basis of segments to a general basis of oriented subgraphs $g_{i}$. The topology of the network is its invariant theory under $G_{\xi}$. The dynamics of the network is the invariant theory of complex vector space under basis transformations $G_{\xi}$ which replace the $n$ segment currents $J^{i}$ by component currents $J^{i *}$ flowing through each segment of the subgraph $g_{i}$ in the direction of its orientation heedless of Kirchoff's law. $J^{i}$ is then the composite effect of the component contributions from each basis subgraph overlapping the segment. The fundamental theorem of electrical networks appears as a reduction to a canonical basis. (Received September 8, 1952.)

25t. H. F. MacNeish: A symmetric method for determining the coefficients of a Fourier series.

$a_{r}, b_{r}$ represent the coefficients of the Fourier series $y=f(x)=\sum_{r=0}^{r=n} a_{r} \cos r x$ $+\sum_{r=1}^{r=n-1} b_{r} \sin r x$, where $f(x)$ is defined by a table of $2 n$ values $\left(x_{i}, y_{i}\right)$, for $i=1$, $2,3, \cdots, 2 n-1$. The Runge solution, called the Runge diagrammatic method, is based on (a) an elaborate diagram involving sums $s_{i}$ and differences $d_{i}$ of the values of $y_{i}$ and further sums and differences of $s_{i}$ and $d_{i}$, etc., taken in an erratic manner, and (b) a set of $2 n$ opaque formulas for $a_{r}$ and $b_{r}$, so that for the simple case $2 n=12,26$ auxiliary letters and 12 formulas are necessary, also (a) and (b) differ unsymmetrically with $n$. (See Scarborough, Numerical mathematical analysis, chap. 17.) In this paper a simple general scheme involving no diagram gives formulas for the coefficients $a_{r}$ and $b_{r}$ for all values of $n$. Only seven symmetric formulas are required involving $2 n$ auxiliary letters $s_{i, i+n}=y_{i}+y_{i+n}$ and $d_{i, i+n}=y_{i}-y_{i+n}$, for which the notation is selfevident. It is assumed that the table of values represents a periodic function for which the period may be taken as $2 \pi$, from which it follows that $x_{i}=\left(180^{\circ} / n\right) i, i=0,1,2$, $\cdots, 2 n-1$. (Received August 26, 1952.)

26t. D. M. Young: On Richardson's method for solving linear systems with positive definite matrices.

For the linear system $\sum_{i=1}^{N} a_{i, j} u_{j}+d_{i}=0(i=1,2, \cdots, N)$ with positive definite matrix, L. F. Richardson (Roy. Soc. Philos. Trans. A 210 (1910)) presented an iterative method $u_{i}^{(n+1)}=u_{i}^{(n)}+\beta_{n+1}\left\{\sum_{j=1}^{N} a_{i, j} u_{j}^{(n)}+d_{i}\right\}(i=1,2, \cdots, N)$, where $u_{1}^{(0)}, u_{2}^{(0)}$, $\cdots, u_{N}^{(0)}$ are arbitrary and where the relaxation factors $\beta_{n+1}$ are to be chosen. Using a theorem on Tschebyscheff polynomials given by Flanders and Shortley (Journal of Applied Physics vol. 21 (1950) pp. 1326-1332) the author has shown that if a good lower bound for the eigenvalues of $\left(a_{i, j}\right)$ is known, the relaxation factors can be chosen so that the average rate of convergence is asymptotically proportional to $R^{1 / 2}$ as $R \rightarrow 0$ where $R$ is the rate of convergence of the method using a fixed $\beta$. For a given integer $m$, the relaxation factors which yield the optimum convergence are given by 
$\beta_{n}=2\left[(b-a) t_{n}-(b+a)\right]^{-1}(n=1,2, \cdots, m)$ where $t_{n}=\cos [(2 n-1) \pi / 2 m]$ and $b$ and $a$ are upper and lower bounds respectively for the eigenvalues of $\left(a_{i, j}\right)$. Although stronger restrictions on $\left(a_{i, j}\right)$ were required to prove the same order-of-magnitude gain in convergence rate for the successive overrelaxation method (Bull. Amer. Math. Soc. Abstract 56-4-322) Richardson's method is not as well adapted for large automatic computing machines and is slower in many cases by a factor of at least two. (Received September 5, 1952.)

\section{GEOMETRY}

\section{L. F. Markus: Orientability of line element fields.}

A continuous field of line elements on a simply-connected differentiable manifold is orientable. A similar statement holds for a regular curve family filling a simplyconnected topological manifold. (Received October 18, 1952.)

\section{Katsumi Nomizu: Group of affine transformations of an affinely connected manifold.}

It is a well known theorem that the group of all isometries of a Riemannian manifold is a Lie group (S. B. Meyers and N. E. Steenrod, Ann. of Math. vol. 40 (1939)). The main purpose of the present paper is to establish a similar theorem for the group of affine transformations of an affinely-connected manifold. Given a manifold $M$ with affine connection $d p=\sum_{i} \theta^{i} e_{i}, d e_{i}=\sum_{i} \theta_{i}^{i} e_{j}$, where $\theta^{i}$ and $\theta_{i}^{i}$ are linear differential forms on the bundle of frames $B$ of $M$ which define the affine connection, a differentiable homeomorphism of $M$ onto itself is called an affine transformation of $M$ if the induced homeomorphism of $B$ leaves the forms $\theta^{i}$ and $\theta_{i}^{i}$ invariant. Theorem. The group $A(M)$ of all affine transformations of $M$, provided with the compact-open topology, is a Lie group. For the proof use is made of a theorem of S. Bochner and D. Montgomery concerning locally compact groups of differentiable transformations (Ann. of Math. vol. 47 (1946)). (Received September 10, 1952.)

\section{9t. Walter Prenowitz: Partially ordered geometries. Preliminary} report.

A partially ordered geometry (p.o. geometry) is defined to be a system consisting of a set $S$ of elements (points) $a, b, c, \cdots$, and a 3-term relation (order) expressed by the notation $(a b c)$ which satisfies the following postulates: 01. $(a a a) ; 02 .(a b c)$ implies $(c b a)$; 03. $\left(a p_{1} q_{1}\right),\left(a p_{2} q_{2}\right)$ imply the existence of $x$ such that $\left(p_{1} x q_{2}\right),\left(p_{2} x q_{1}\right) ; 04$. $\left(a p_{1} q_{1}\right),\left(p_{2} a q_{2}\right)$ imply the existence of $a^{\prime}$ such that $\left(a^{\prime} p_{1} q_{2}\right),\left(p_{2} a^{\prime} q_{1}\right) ; 05$. For any $a, b$ there exists $x$ such that $(a b x)$. A p.o. geometry is simply ordered if it satisfies 06 . $(a b x),(a b y), x \neq y$ imply $(a x y)$ or $(a y x)$. The notion p.o. geometry is introduced with the object of unifying the theory of classical geometries and their generalizations. Any descriptive geometry becomes a p.o. geometry if we define $(a b c)$ to mean either $b$ is an interior point of segment $a c$ or $a=b=c$. A spherical geometry may be converted into a p.o. geometry by the adjunction of an "ideal" point bearing appropriate order relations to the given points. Any projective geometry also can be converted into a p.o. geometry by adjoining properly an "ideal" point. Any Boolean algebra becomes a p.o. geometry if $(a b c)$ is defined to mean $a \bigcap c \leqq b \leqq a \cup_{c}$. There exist p.o. geometries which are not simply ordered and whose points and "lines" form an affine geometry (Prenowitz, Partially ordered fields and geometries, Amer. Math. Monthly vol. 53 (1946) pp. 439-449). (Received September 15, 1952.) 


\section{LOGIC AND Foundations}

30. M. D. Davis: Note on a conjecture of Post.

$\left|\pi_{\alpha}\right|,\left|\bar{\pi}_{\alpha}\right|, \alpha<\omega^{2}$, are defined to be sequences of quantifiers as follows: $\left|\pi_{1}\right|$ is $\left(E x_{1}\right),\left|\bar{\pi}_{1}\right|$ is $\left(x_{1}\right),\left|\pi_{\alpha+1}\right|$ is $\left(E x_{\alpha+1}\right)\left|\bar{\pi}_{\alpha}\right|,\left|\bar{\pi}_{\alpha+1}\right|$ is $\left(x_{\alpha+1}\right)\left|\pi_{\alpha}\right|,\left|\pi_{\omega p}\right|$ is $\left(E y_{p}\right)\left(k_{p}\right)_{k_{p} \leqq y_{p}}$ $\left(E n_{p}\right)\left|\pi_{\omega(p-1)+n_{p}}\right|$, and $\left|\bar{\pi}_{\omega p}\right|$ is $\left(y_{p}\right)\left(E k_{p}\right)_{k_{p} \leqq y_{p}}\left(n_{p}\right)\left|\bar{\pi}_{\omega(p-1)+n_{p}}\right|$. Let the set of integers $K_{\alpha}, \alpha<\omega^{2}$, be defined by taking $K_{0}$ to be empty, and setting $K_{\alpha+1}=\left(K_{\alpha}\right)^{\prime}, K_{\omega p}$ $=\left\{x \mid(x)_{1} \in K_{\omega(p-1)+(x)_{2}}\right\}^{\prime}$. (The ' notation follows Post's abstract, Bull. Amer. Math. Soc. vol. 54-7-269.) For $1 \leqq \alpha<\omega^{2}$, let $\boldsymbol{P}_{\alpha}$ be the class of all predicates which are manyone reducible to $K_{\alpha} ; \boldsymbol{Q}_{\alpha}$ the class of negations of members of $\boldsymbol{P}_{\alpha}$. Then, it is shown that a predicate $W$ belongs to $\boldsymbol{P}_{\alpha}\left(\boldsymbol{Q}_{\alpha}\right)$ if and only if there is a recursive predicate $N$ [some of whose variables may range over the set of all finite sequences of natural numbers] such that $W \equiv\left|\pi_{\alpha}\right| N\left(W \equiv\left|\bar{\pi}_{\alpha}\right| N\right)$. This extends a theorem of Post (loc. cit.) into the transfinite, as conjectured by him, making use of an extension of Kleene's hierarchy of predicates developed in the author's dissertation (Princeton, 1950). (Received October 16, 1952.)

\section{Hartley Rogers, Jr.: Some results on definability and decidability in elementary theories. I.}

In Journal of Symbolic Logic vol. 16 (1951) pp. 239-240, Church and Quine show that a general binary relation is definable from a symmetric relation in the sense that an expression of quantification theory exists, in one binary function variable and two free individual variables, such that as symmetric relations are assigned to the function variable, the value of the entire expression runs through all binary relations. The general decision problem for quantification theory thus reduces to that for a single symmetric relation. As intermediate degrees of undecidability may possibly exist, this is at present somewhat stronger than an undecidability result alone. Using relation-sets - elementary theory models which are values for higher variables in thirdorder functional calculus-the writer develops a theoretical framework for results of this general kind. The theory accommodates results somewhat beyond the immediate definability method of the Church-Quine paper. The theory is applied to obtain reduction-undecidability-definability for the elementary theory of a single binary disjoint relation (domain and co-domain disjoint), of a single symmetric reflexive relation, and of a single transitive reflexive relation. Reduction-undecidability results are obtained for the cases of two equivalences, one equivalence and one total order, and two total orders. (Received August 6, 1952.)

\section{2t. Hartley Rogers, Jr.: Some results on definability and decidabil-} ity in elementary theories. II.

A decision method for the elementary theory of a single equivalence is known, also for monadic quantification theory with identity, i.e., the elementary theory of a single equivalence $R$ and any number of singulary relations $F_{i}$ which are well-defined with respect to that equivalence $\left(R(x, y) \supset_{x y} \cdot F_{i}(x) \supset F_{i}(y)\right.$ is true). These results are here extended by deriving decision methods for the elementary theory of a single symmetric and transitive relation, and for the elementary theory of a single equivalence together with any number of singulary relations, well-defined or not. By the elementary theory of rectangular arrays we shall mean that theory obtained when we consider matrix arrays of 0 's and 1's, use two binary constants $C$ and $R$ interpreted as "in the same column as" and "in the same row as," consider occurrences of 1 's as 
individuals, and construct formulas within quantification theory. Results of this paper and the preceding paper are used together to show that the theories of $m \times$ finite, $m \times \infty$, as well as $m \times n$ arrays are decidable, while the theory for $\infty \times \infty$ arrays is undecidable though axiomatizable. The theories for finite $\times$ finite and finite $\times \infty$ arrays are shown equivalent, though decision and axiomatizability here remain undetermined. (Received August 6, 1952.)

\section{Statistics AND Probability}

33t. Herbert Robbins: On the equidistribution of the sequence of cumulative sums of independent random variables.

Let $\left\{X_{n}\right\}$ be independent random variables with a common distribution $F$ and let $S_{n}=X_{1}+\cdots+X_{n}$. The author is interested in the general problem of proving that, under certain hypotheses on $F$, the sequence $\left\{S_{n}\right\}$ is with probability 1 "equidistributed" (i.e. "uniformly dense") on the line $-\infty<x<\infty$ in some sense to be made precise. For example, if $g(x)$ is any almost periodic function, then, with probability 1 , $\lim _{n \rightarrow \infty} n^{-1} g\left(S_{j}\right)=\bar{g}=$ mean value of $g(x)$; also, with probability 1 the sequence $\left\{S_{n}\right\}$ is equidistributed in the usual sense modulo $I$ simultaneously for all finite intervals $I$. These results hold for any nonlattice distribution $F$. Deeper equidistribution properties hold, involving limits of ratios $\sum_{1}^{n} g\left(S_{j}\right) / \sum_{1}^{n} h\left(S_{j}\right)$ for a wider class of functions $g(x), h(x)$ (e.g. the characteristic functions of any two finite intervals) provided $F$ is suitably restricted. (Received September 26, 1952.)

\section{TOPOLOGY}

\section{Alice B. Dickinson: Compactness conditions and uniform struc- tures.}

Let a uniform structure which is less fine than all the other uniform structures compatible with the topology of the space be called the crude structure of the space. In a locally compact space the uniform structure induced by the uniquely defined onepoint compactification is the crude structure. A unique uniform structure implies a unique compactification, in particular the one-point compactification of a locally compact space. The proof of this second theorem demonstrates a method of construction of distinct uniform structures. If a space has a unique uniform structure it is countably compact. A paracompact space is complete with respect to its universal structure. This last theorem answers questions raised by Andre Weil (Sur les espaces à structure uniforme et sur la topologie générale, Actualités Scientifiques et Industrielles, no. 551, Paris, Hermann, 1937) and J. Dieudonné (Une généralisation des espaces compacts, J. Math. Pures Appl. vol. 23 (1944) pp. 65-76). Relative to paracompactness, it is shown that the three properties, paracompactness, superior compactness from $\aleph_{1}$, and the property that every covering has a star finite refinement, are equivalent in a space which is locally countably compact and has at most a countable number of quasi components. (Received July 14, 1952.)

\section{5t. W. H. Gottschalk: Intersection and closure.}

Let $X$ be a uniform space and let $A, B \subset X$. The sets $A$ and $B$ are uniformly separable provided that if $U$ is a uniform neighborhood of $A \cap B$, then $A-U$ and $B-U$ have disjoint uniform neighborhoods. It is proved that: (1) If $A$ and $B$ are uniformly separable, then $\mathrm{Cl}(A \cap B)=\mathrm{Cl}(A) \bigcap \mathrm{Cl}(B)$; (2) If $A$ or $B$ is conditionally compact and if $Y$ is a dense subset of $X$ such that $A \cup B \subset Y$, then $\mathrm{Cl}(A \cap B)=\mathrm{Cl}(A) \cap \mathrm{Cl}(B)$ 
(closures in $X$ ) if and only if $A$ and $B$ are uniformly separable in $Y$. (Received August 4, 1952.)

\title{
36t. G. D. Mostow: Factor spaces of solvable groups.
}

Definition. A solvmanifold is a manifold in which a solvable Lie group of transformations acts transitively. In the special case that the group is nilpotent, one has a nilmanifold (introduced by Malcev, A.M.S. Translation, no. 39). The two-dimensional solvmanifolds are the plane, cylinder, torus, Klein bottle, Mobius band. Theorem 1 . Two compact solvmanifolds having isomorphic fundamental groups are homeomorphic (Bull. Amer. Math. Soc. Abstract 58-2-261). Corollary. A compact solvmanifold whose fundamental group is abelian is homeomorphic to a toroid. Theorem 2. Any solvmanifold is regularly covered a finite number of times by a direct product of a compact solvmanifold and a euclidean space. The covering group is abelian. In certain special cases, one can assert that the covering space is univalent. As a special case one obtains: Corollary (Chevalley). The factor space of a solvable Lie group by a connected subgroup is homeomorphic to a direct product of circles and straight lines. (Received August 4, 1952.)

\author{
L. W. Cohen, \\ Associate Secretary
}

